\title{
Intertrial interval shift effects on discrimination reversal: Motivational and associative control by internal stimuli
}

\author{
STEVEN J. HAGGBLOOM \\ Arkansas State University, State University, Arkansas 72467
}

\begin{abstract}
Four groups of eight rats each were trained on a successive brightness differential conditioning problem under a +- trial sequence in which all daily trials to the positive $(\mathrm{S}+)$ stimulus preceded those to the negative (S-) stimulus. The groups composed a 2 by 2 factorial combination of intertrial intervals of 1 and $15 \mathrm{~min}$ in Phases 1 and 2. The four groups, identified by Phase 1 and Phase 2 intertrial interval, were Groups 1/1, 1/15, 15/1, and 15/15. In Phase 2, brightness cues were reversed for each group. The original discrimination was acquired more rapidly by Groups $1 / 1$ and $1 / 15$ than by Groups $15 / 1$ and 15/15. Reversal learning was best in Group 1/1, worst in Group 1/15, and intermediate in Groups 15/15 and 15/1. The results were discussed in terms of their implications for motivational and associative control over discriminative behavior by internal, reward-produced cues.
\end{abstract}

Groups given all of their daily trials to the positive (S+) stimulus preceding their trials to the negative (S-) stimulus ( +- sequence) in successive brightness differential conditioning learn to respond appropriately to the nominally relevant brightness cues, such as a black vs. a white runway, more rapidly than groups given an intermixed (+- +) sequence of S+ and S- trials (Haggbloom, 1978; Haggbloom \& Tillman, 1980). Under the +sequence, brightness and internal, reward-produced cues, such as memories of reward $\left(\mathrm{S}^{\mathrm{R}}\right)$ and nonreward $\left(\mathrm{S}^{\mathrm{N}}\right)$, are concomitantly relevant and both sets of cues gain associative control over discriminative behavior (Haggbloom, 1981; Haggbloom \& Tillman, 1980). Except for the transition from $S+$ to $S-, S^{R}$ produced on one trial predicts reward on the next trial and $S^{N}$ produced on one trial always predicts nonreward on the next trial.

In addition to promoting correct discriminative behavior by functioning as additional relevant discriminanda and exercising associative control over responding under the +- sequence, internal cues, especially $\mathrm{S}^{\mathbf{N}}$, quite likely have a nonassociative, motivational, or emotional effect on responding. Nonreward clearly has emotional consequences that can produce slower responding on a subsequent trial (e.g., Capaldi, Berg, \& Sparling, 1971; Gray, 1969).

The purpose of the present experiment was to assess the relative contribution of associative and motivational control by internal cues to facilitated discrimination learning under the +- sequence. The experiment employed a reversal of brightness cues coupled with a shift in intertrial interval (ITI). The reversal of bright-

This experiment was supported by a Faculty Research Grant from Arkansas State University. ness cues under conditions in which internal cues continue to predict reward outcome as before (i.e., employing the +- sequence in both original learning and reversal learning) has proved to be a sensitive test of the control exercised by internal cues (Haggbloom, 1981; Haggbloom \& Tillman, 1980). The behavioral effects of ITI in discrimination learning (e.g., Haggbloom, 1978, 1979), as in partial reinforcement situations (Capaldi et al., 1971; Capaldi \& Haggbloom, 1975), are mediated by ITI-dependent changes in internal stimulus control over responding. ITI shifts have proved to be useful in separating motivational and associative aspects of that control (Capaldi et al., 1971; Haggbloom, 1979).

Four groups received Phase 1 training on a successive brightness differential conditioning problem under the +- sequence. In Phase 2, brightness cues were reversed, but the +- sequence remained in effect. Groups $1 / 1$ and $15 / 15$ received the same ITI in both phases of training; respectively, they received ITIs of 1 and $15 \mathrm{~min}$. Group 1/15 was shifted from a 1-min ITI in Phase 1 to a 15-min ITI in Phase 2; the opposite arrangement held for Group 15/1.

\section{METHOD}

\section{Subjects}

The subjects were 32 male rats, 90 days old at the beginning of training, bred in the laboratory from Holtzman stock.

\section{Apparatus}

The apparatus consisted of two parallel straight alleys, $105 \mathrm{~cm}$ long $\times 9 \mathrm{~cm}$ high and wide. The walls and floor of one alley were painted white, and the walls and floor of the other alley were painted black. The last $25 \mathrm{~cm}$ of each alley constituted a goalbox separated from the rest of the alley by a manually operated guillotine door. The doors and goalboxes were painted the same color as the alley in which they were located. Each goalbox contained an unpainted wooden goal cup. 
A gray startbox, $9 \mathrm{~cm}$ high and wide $\times 25 \mathrm{~cm}$ long, could be aligned to permit entry into one alley or the other. The startbox had a gray, manually operated guillotine door. Start times were recorded from the opening of the startbox door, which triggered a .01-sec clock, to a point $32 \mathrm{~cm}$ into the alley. Run and goal times were recorded over the next 40 and $30 \mathrm{~cm}$, respectively, of the alley. The offset of the first clock and the operation of the run and goal clocks were controlled by photoelectric circuitry.

\section{Procedure}

Two weeks prior to the 1st day of experimental training, all food was removed from each rat's cage and a daily ration of $12 \mathrm{~g}$ of Lab Chow was begun. The rats had free access to water at all times. On Days 12-14, the rats were handled in squads of four for $4 \mathrm{~min} / \mathrm{squad}$. After being handled on those days, the rats were fed $1045-\mathrm{mg}$ Noyes food pellets in their home cage. Eight rats were randomly assigned to each of four groups defined by the factorial combination of Phase 1 ITI (1 vs. $15 \mathrm{~min})$ and Phase 2 ITI ( 1 vs. $15 \mathrm{~min})$. The four groups, identified by Phase 1 and Phase 2 ITI, were Groups $1 / 1,1 / 15,15 / 1$, and $15 / 15$.

In Phase 1 , each rat received two $S+$ trials followed by two $S$ - trials on each of 10 days. On each of the 4 days of Phase 2, each group continued to receive two daily $S+$ trials followed by two $S$ - trials. For each group, the brightness cues serving as $S+$ and $S-$ were reversed from Phase 1 to Phase 2. Appropriate counterbalancing of brightness cues serving as $\mathrm{S}+$ and $\mathrm{S}-$ was employed.

On S+ trials, the rats were removed from the goalbox after the times were recorded (after approximately $10 \mathrm{sec}$ ) unless the reward had not yet been consumed. All reinforcements consisted of $1045-\mathrm{mg}$ Noyes pellets. On S- trials, the rats were confined to the unbaited goalbox for $20 \mathrm{sec}$. A trial was begun by placing a rat in the startbox and opening the startbox door after approximately $3 \mathrm{sec}$, regardless of the rat's orientation. The rats were run in squads of four containing one rat from each group. The order in which trials were administered within a squad varied randomly from day to day, and the order of successive squads was held constant across days. The ITI was 3-4 min. A maximum time of $30 \mathrm{sec}$ was allowed in each alley section. If $30 \mathrm{sec}$ was exceeded in any alley section, the additional time was added to the time score of the next section forward. If the rat did not enter the goalbox within $90 \mathrm{sec}$, it was placed in the goalbox.

\section{RESULTS}

Time scores for each section of the alley were summed for each rat for each trial to obtain a total time. All times were converted to speeds in centimeters per second. Difference scores were obtained by subtracting the mean daily $S-$ speed from the mean daily $S+$ speed for each rat. Only analyses applied to total speeds and difference scores based on total speeds are reported here.

In Phase 1 (data not shown), Groups $1 / 1$ and $1 / 15$ showed comparable discriminative behavior and better discriminative behavior than Groups $15 / 1$ and 15/15, which did not differ from each other. A 2 (Phase 1 ITI) by 2 (Phase 2 ITI) by 2 (brightness) between-groups analysis of variance (ANOVA) applied to difference scores on the last day of Phase 1 yielded a significant Phase 1 ITI effect $[F(1,24)=4.47, p<.05]$, but no Phase 2 ITI effect $[F(1,24)=1.24]$ or Phase 1 ITI by Phase 2 ITI interaction $(F<1)$. An additional ANOVA and subsequent simple effects of dicriminanda at each group showed that $\mathrm{S}+$ speeds were reliably faster than $\mathrm{S}-$ speeds at the end of Phase 1 in each of the four groups [smallest $F(1,24)=11.43, p<.01$, for Group 15/1] .

Figure 1 shows the mean difference between speeds in $S+$ and $S-$ for each group on each of the 4 days of Phase 2. As can be seen in Figure 1, the reversal of brightness cues produced a marked disruption of discriminative behavior in each group. Acquisition of the reversal discrimination was best in Group $1 / 1$, worst in Group $1 / 15$, and intermediate in Groups $15 / 1$ and $15 / 15$. Two ANOVAs, identical to the one applied to difference scores on the last day of Phase 1, were applied to difference scores on the first and last days of Phase 2. There were no differences in discriminative behavior on Day 1 of Phase 2 due to either Phase 1 or Phase 2 ITI or their interaction [largest $F(1,24)=2.30$ for the effect of Phase 2 ITI]. The ANOVA applied to Day 4 yielded a reliable Phase 1 ITI by Phase 2 ITI interaction $[\mathrm{F}(1,24)=8.55, \mathrm{p}<.01]$.

Simple effects of Phase 1 ITI at Phase 2 ITI showed that the difference between Groups $15 / 15$ and $1 / 15$ was reliable $[F(1,24)=5.85, p<.05]$, but that the difference between Groups $1 / 1$ and $15 / 1$ did not reach a conventional level of significance $[F(1,24)=2.95]$. Simple effects of Phase 2 ITI at Phase 1 ITI showed that there was no difference between Groups 15/1 and $15 / 15(\mathrm{~F}<1)$. The difference between Groups $1 / 1$ and $1 / 15$ was highly reliable $[F(1,24)=19.26, p<.01]$.

Additional ANOVAs and subsequent simple effects of discriminanda for each group on Days 2-4 of Phase 2 showed that $\mathrm{S}+$ speeds were faster than $\mathrm{S}-$ speeds in Groups $1 / 1 \quad[\mathrm{~F}(1,24)=7.86, \mathrm{p}<.01]$ and $15 / 15$ $[F(1,24)=4.87, p<.05]$ by Day 2 , and in Group $15 / 1$ by Day $3[F(1,24)=6.79, p<.05]$. Group $1 / 15$ did not run reliably faster in S+ than in $S-$ on any day of Phase 2 (all Fs $<1$ ).

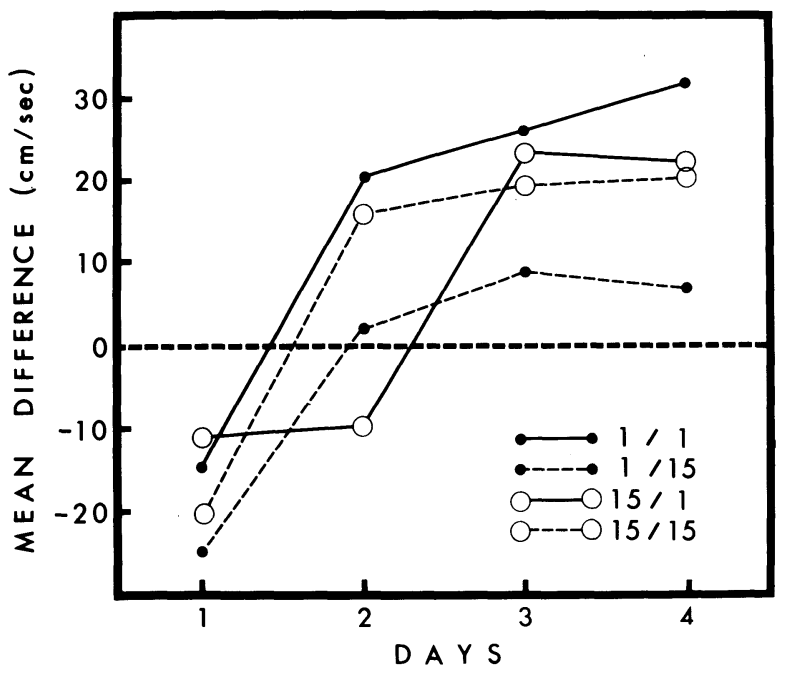

Figure 1. Mean difference between $S+$ and $S$ - speeds for each of the four groups on each day of Phase 2 . 


\section{DISCUSSION}

In Phase 1, acquisition of a successive go/no-go discrimination occurred more rapidly in Groups $1 / 1$ and $1 / 15$, given a 1-min ITI, than in Groups 15/1 and 15/15, given a 15-min ITI. This effect of ITI on acquisition of an original discrimination is fully in agreement with previously reported effects of ITI in discrimination learning situations in which internal cues were relevant discriminanda (Haggbloom, 1978, 1979).

The results obtained here in Phase 2 are consistent with the view that internal cues exercise both motivational and associative control over discriminative responding under the +- schedule. Acquisition of the reversed discrimination occurred most readily in Group 1/1, in which there was presumably no change in associative control by internal cues, and motivational control also favored rapid discrimination learning. Performance on the reversal problem was poorest in Group $1 / 15$. The increase in ITI from Phase 1 to Phase 2 in Group $1 / 15$ was expected to result in a loss of both motivational and associative control by internal cues. Associative control was expected to decrease because control in Phảse 1 was acquired by an ITI-specific (1-min) value of internal cues and the ITI shift introduced a new and unconditioned ITI-specific value of those cues. Haggbloom (1979) reported that rats can discriminate between (2- and 30-min) ITI-specific values of internal cues and that such cues can exercise associative control over discriminative responding. Group 1/15 was expected to suffer a loss of motivational control on $\mathrm{S}-$ trials as a consequence of the increase in ITI since, as ITI increases, the emotional, frustrative reaction to nonreward dissipates (e.g., Capaldi et al., 1971). Thus, the different rate of reversal learning between Groups $1 / 1$ and $1 / 15$ reflects a difference in both associative and motivational control by internal cues.

In Group $15 / 15$, as in Group $1 / 1$, there was no change from Phase 1 to Phase 2 in the associative control exercised by internal cues, since the same $15-\mathrm{min}$ value of internal cues controlled behavior in both phases. However, motivational control on $\mathrm{S}-$ trials would be less in Group $15 / 15$ than in Group 1/1. The difference in rate of reversal learning between Groups $15 / 15$ and $1 / 1$, then, presumably reflects a difference in motivational control by internal cues.

Finally, the difference in rate of reversal learning between Groups $1 / 1$ and $15 / 1$ presumably reflects a difference in associa- tive control by internal cues. A loss of associative control by internal cues was expected in Group 15/1, as it was in Group $1 / 15$, as a consequence of the ITI shift and the introduction of new and unconditioned values of internal cues. Motivational control on $\mathrm{S}-$ trials, however, would be increased in Phase 2 in Group $15 / 1$ and would favor more rapid reversal learning. That Group $15 / 1$ lagged only slightly behind Group $15 / 15$ in reversal learning suggests that associative and motivational control by internal cues contributed about equally to the control of discriminative behavior under the +- sequence.

On a more general level, the present results are consistent with prior results (Haggbloom, 1978, 1981; Haggbloom \& Tillman, 1980), indicating that internal cues exercise substantial control over behavior in brightness differential conditioning and that ITI effects on discrimination learning are mediated by ITI-dependent changes in internal stimulus control (Haggbloom, 1978, 1979).

\section{REFERENCES}

Capaldi, E. J., Berg, R. F., \& Sparling, D. L. Trial spacing and emotionality in the rat. Journal of Comparative and Physiological Psychology, 1971, 76, 290-299.

Capaldi, E. J., \& Haggbloom, S. J. Response events as well as goal events as sources of animal memory. Animal Learning \& Behavior, 1975, 3, 1-10.

Gray, J. A. Sodium amobarbital and the effects of frustrative nonreward. Journal of Comparative and Physiological Psychology, 1969, 69, 55-64.

HAGGBLOOM, S. J. Intertrial interval effects on internal stimulus control of behavior in brightness differential conditioning. Learning and Motivation, 1978, 9, 347-358.

HagGBloom, S. J. The differential reinforcement of rewardproduced and response-produced stimuli. Learning and Motivation, 1979, 10, 364-381.

HagGBloom, S. J. Blocking in successive differential conditioning: Prior acquisition of control by internal cues blocks the acquisition of control by brightness. Learning and Motivation, 1981, 12, 485-508.

Haggbloom, S. J., \& Tillman, D. J. Sequential effects on discrimination reversal. Learning and Motivation, 1980, 11, 318-338.

(Received for publication September 13, 1982.) 